\title{
Narration in Qualified Media Types
}

\begin{abstract}
This chapter illuminates and roughly summarizes some vital concepts and ideas of the whole treatise. Narration in four diverse qualified media types is explored: painting, instrumental music, mathematical equations, and guided tours. These qualified media types are grounded on partly very dissimilar basic media types, which also makes it possible to highlight the fundamental importance of media modalities for narration. The four investigations also illustrate how historically and socially qualified media types establish conventions that facilitate narration. Overall, the four investigations elucidate the usefulness of the theoretical framework developed in the treatise and highlight the media similarities and differences that make narration a profoundly transmedial but media-dependent phenomenon.
\end{abstract}

Keywords Transmedial narration $\bullet$ Qualified media $\bullet$ Painting • Instrumental music $\bullet$ Mathematical equations $\bullet$ Guided tours

In selecting qualified media types I have avoided those that have been most popular in narratological research, such as various forms of literature, film, and comics. To push the transmedial perspective, I chose to inspect media types that challenge overly narrow limits of narration. I begin with two already partly explored artistic qualified media types-painting and instrumental music- and end with two unexplored 'non-artistic' qualified

(C) The Author(s) 2019

L. Elleström, Transmedial Narration, https://doi.org/10.1007/978-3-030-01294-6_10 
media types: mathematical equations and guided tours. As my goal is to highlight the broad transmedial applicability of essential narrative concepts, the four investigations will be structured similarly, while also revealing in some detail the media-specific differences.

\section{PAINTING}

There is a long history of research on the issue of narration in painting. Because painting is normally understood as a qualified form of the basic media type of still image, the results of this research can, to some extent, be extended to narration in still images in general. Paintings are still images in that they are usually expected to be solid (and generally inorganic), two-dimensionally spatial and non-temporal, visual, and predominantly iconic. Being predominantly iconic means that the visual surface is at an initial stage primarily understood to resemble the objects that they represent, although the represented objects are not necessarily concrete and visual. Paintings, like other forms of media products, may be cross-modally iconic, meaning that the iconicity is based on similarities among, for instance, different sense perceptions, and between concrete and abstract entities (Elleström 2017). This means that so-called abstract or nonfigurative art may also be iconic, representing objects such as rhythms and patterns of sound or tactile sensations, or more abstract notions such as clashes, speed, harmony, chaos, or rest.

Although the capacity to hold cross-modal iconicity facilitates transmediation among different media types in general, and also transmediation of narratives, narration in painting faces one obstacle: because the materiality of still images does not evolve in time, painting is a static media type. As already noted, this puts narration to the test because non-temporal media products do not readily represent temporal relationships among events. However, as I concluded earlier, this does not eliminate the narrative capacity of still images and hence paintings.

This is not the forum for extensive elaborations on how painting as a qualified media type can be delineated. One crucial point of the concept of qualified medium is that qualified media types are bound to vary and change, which means that I can only make approximations here. If one initially circumscribes paintings as still images that are handcrafted by people using various techniques for applying color to a surface, one may note that there is a multitude of specific traditions and functions of paintings in various cultures. Of course, no one really knows precisely how ancient 
people used and appreciated for instance rock-paintings, and still today paintings have rather different functions depending on culture and other contexts. Paintings may be expected to have, say, religious, aesthetic, educational, or practical uses, or a mixture of them all. For more thorough investigations, therefore, it is probably more useful to think in terms of several interrelated rather than one single qualified medium of painting.

As painting is such a broad media category, there are virtually no limits to the kinds of events that a painting may depict. Anything that can be represented by visual still images may be harbored within painting conceived as an all-embracing qualified medium. Naturally, criteria for what kinds of events qualify for proper painting vary considerably depending on time and culture. For instance, at a certain moment in a certain place, only events from Christian history and mythology might be accepted. Additionally, there are genres of painting that further qualify what kinds of existents, states, and events should be included. Therefore, genres may appropriately be called submedia.

Some paintings, such as so-called still lifes, represent only states, such as food lying on a table. Although it is not impossible to narrativize such states, there is a huge difference between still lifes and paintings depicting myriad people eating, fighting, or playing. In the latter case, both concrete events such as jumping and falling and abstract events such as winning and losing may be directly represented. Given a sufficient amount of events, they may also be hierarchized by the actual communicatee on the basis of the appearance of the media product - the painting. On one hand, represented events may be given a dominant role because of, say, large size, foreground location, or central position on the two-dimensional surface of the painting. On the other hand, represented events may be perceived to be principal because of their existential weight; someone dying in the background may well be a more essential event than someone having a beer in the foreground.

Given the capacity of paintings to represent a multitude of concrete and abstract events, both actions and occurrences, it is fair to say that paintings, photographs, and other still images have "narrative implications" (Kafalenos 1996). It is also clear that painting and other qualified media types based on visual still images have, for thousands of years, developed conventions that enhance narration. In semiotic terms, this adds a symbolic element to the predominantly iconic medium of painting. Research in the historical development of ancient qualified media types such as epic literature, vase painting, and illustrated written religious texts have dis- 
cerned three methods of realizing already known literary narratives in visual art (Wickhoff 1895: 8-9; Weitzmann 1947: 12-33). These categories are echoed in later and more general research on transmedial narration (such as Kibédi Varga 1988; Wolf 2003: 189-191). Formulated in my own terminology and with the addition of some reflections, narration is achieved through the three following means:

- One single confined two-dimensional space in which one event is directly represented. This event calls other temporally interrelated events to mind so that they can together form a narrative. As stated in Chap. 7, there are at least two ways of achieving narration in this way. Either collateral experience in the form of general knowledge of the natural and cultural world is called forth to provide additional events, or collateral experience in the form of specific knowledge of an already existing narrative complements the directly represented event so that other temporally interrelated events can be added.

- One single confined two-dimensional space in which several events are directly represented. These events can be sorted temporally by the perceiver and form a narrative. As there are conventions for this kind of interpretation in painting, beholders are invited to construe such temporal interrelations.

- Several confined two-dimensional spaces in which one event per space is directly represented. These spaces are supposed to be perceived sequentially. In other words, narration is enhanced through a convention of sequential decoding in analogy with the convention of reading written verbal texts.

In principle, one could add a fourth possibility (Kibédi Varga 1988); namely, several confined two-dimensional spaces in which several events per space are directly represented. These spaces should also be perceived sequentially.

There are clearly many ways of representing several temporally interrelated events in painting and these may include temporal differences between narratives and stories (Genette 1980 [1972]: 33-160). A conventionally decoded sequence of events may form a narrative in which the order of events differs from the order in the story. Also, paintings that are not sequentially arranged can hold a temporal difference between narrative and story in terms of frequency: a single confined two-dimensional space in which several events are represented may represent what is per- 
ceived to be the same event several times (meaning at different places), although it only happens once in the story.

Thus, narration in painting profits from habitually grounded ways of ordering represented events or even perceiving them in a certain order. In addition, painting often includes another symbolic element: visual verbal titles. These may clearly add to the overall narration by representing interrelated events that, for instance, specify or augment the events represented by the visual icons. Werner Wolf emphasized the importance of captions pointing to "cultural scripts" and thus enhancing narration (Wolf 2003: 191).

Given that there are several interrelated media types of painting, rather than one single type, the issue of expected truthfulness in painting is anything but straightforward. However, it is important to emphasize that even though painting is generally categorized among other art forms as some sort of 'fiction', successful painting is certainly not believed to lack truthfulness. Although indexicality in painting can clearly be understood in terms of texture and patterns of color having real connections to how the artist's tools mechanically formed the surface-which is relevant when establishing who actually created a painting-mental contiguity is of more immediate interest for most perceivers of painting. In terms of mental contiguity, the way in which things have actually been experienced by minds exposes other forms of truthfulness to the many kinds of mental and material objects in the perceived actual sphere. Obviously, different types of painting are expected to represent different forms of indexical objects understood in terms of, say, universals or particulars and wholes or details. Whereas paintings of the crucifixion of Christ should be truthful to how humans generally suffer or exult, paintings of specific historical events are supposed to be truthful to the appearance of individuals. Whereas impressionistic works are expected to be truthful to how the whole visual area is perceived, so-called trompe l'oeil paintings must be truthful to both the whole and all of its details. All these forms of truthfulness may be part of painted narratives.

\section{Instrumental Music}

Narratological research, including studies in transmedial narration, often ignores the rather extensive research in musical narration. Márta Grabócz (2008) provides an overview of the many ideas about narration in music and the subject is somewhat controversial. Malgorzata Pawlowska (2014) 
offers a good summary of the shifting opinions on the existence of narration in music. The hesitation to embrace the idea of music being potentially narrative does not so much concern music including song, or having words integrated in other ways, as music (almost) completely lacking verbal components-which I refer to here as instrumental music. As the concept of narration is still strongly associated with verbal media types, some scholars consider music that has perhaps at most an abstract title as the only verbal constituent to be non-narrative, almost by definition. I will refrain from explicitly arguing against these views, which are often variously formulated in nuanced ways, and instead pick up some rewarding suggestions of how to conceptualize narration in music. My contention is that it is both unproblematic and constructive to consider some instrumental music narrative.

The presemiotic basic media traits of music are fairly clear-cut. Materially, music simply consists of vibrating air. These vibrations are temporal because they change as time passes; all music is perceived within a certain time frame. Music may also be three-dimensionally spatial to a certain extent. If the point of departure for the vibrations is one single location, like a guitar, the vibrations are spread in the room in a fairly symmetrical way, making us perceive the music as one sensation, albeit distributed in space. If the points of departure for the vibrations are several locations - such as a guitar, a flute, and a set of drums - the vibrations are spread throughout the room so that one may discern spatial differences. This three-dimensionality of music is more diffuse than the threedimensionality of, say, a gesturing body, but concrete enough to contribute to the signification of music. Although music may be defined to include the visual perception of for instance performing musicians, I delimit it here to the auditory.

It is perhaps more controversial to state that the dominant semiotic mode of instrumental music is iconicity. It would require a large detour from the primary objective of this treatise to explain the many conflicting ideas about musical signification, or more generally musical meaning. Therefore, here I only suggest that musical signs are auditory signs largely referring to motions, emotions, bodily experiences, and cognitive structures on the ground of similarity. Melodies, pitches, intervals, rhythms, dynamics, sound qualities, and the way they together form ever-developing structures represent similar structures in the externally perceived common world around us, in the internally perceived personal world within us, and in the emotionally and cognitively experienced mental domain within us. 
Representing emotions, for instance, is not the same as evoking emotions: a listener can perceive that a piece of music represents happiness without becoming happy herself.

From a presemiotic point of view, instrumental music is no doubt suitable for representing temporality and therefore potentially for narrating because all musical media products evolve in time. The challenge of narration in instrumental music concerns the semiotic rather than the spatiotemporal modality, mainly because of the absence of well-developed symbolicity in the form of verbal language.

Instrumental music, understood as a qualified medium and not just any form of communicative non-verbal sound, is generally expected to have some sort of aesthetic qualities, in a broad sense (including lighter as well as more highbrowed aesthetics). Because instrumental music, just like painting, is such a broad category, a more detailed study would have to make distinctions among an abundance of all forms of submedia, genres, in order to give a representative picture. Like all qualified media types, instrumental music is qualified differently in different periods, cultures, and subcultures. Since virtually all research on narration in music that I am aware of concerns Western classical (tonal) music, this will also be my point of departure-not because I think it is necessarily the best illustration of narration in instrumental music but because it makes it easier to incorporate existing research in my argumentation.

Having swiftly established that the representation of temporality poses no problem for instrumental music, the remaining core question for narration concerns the representation of events. It is evident that instrumental music does not freely represent events that are as clearly and intersubjectively definable as many events represented by language-based media types using advanced symbol systems, media types using visual icons (photography, dance), or both (television news, comics). In those media types, events can often also be connected to concrete existents, which make them more palpable. In instrumental music, events must primarily be understood in terms of changes in tempo, dynamics, sound qualities, keys, and so forth. Events such as these, and also the existents that they are grounded on, are abstract rather than concrete. Nevertheless, existents and events represented by auditory icons can certainly be perceived as having meaningful interrelationships.

Anthony Newcomb discussed this in terms of temporally ordered musical events where the listener experiences directedness, continuation, and potential (Newcomb 1987). Also, Eero Tarasti treated narration in music 
in terms of temporally emerging tensions and forces among events; he additionally reasoned more specifically, in terms of musical entities such as "actants" or "actors" that fulfill different functions in the musical narrative (Tarasti 1994). Susan McClary emphasized relations among themes and keys and the possibility of thinking about tunes as "protagonists", together forming tensions in the temporal development and possibly disruptions of expectations (McClary 1994, 1997; cf. Seaton 2005). Broadening the scope, Vera Micznik wrote about musical narration in a more far-reaching sense, beyond directions, forces, and tensions among abstract events represented iconically (Micznik 2001). She also considered connotations (which I would translate as indexicality) and sense created by musical conventions (which I would call symbolicity). Although limited in their scope compared to those extensive systems of symbols that we call verbal languages, such musical symbols also connect more directly to the exterior and can hence represent more concrete existents and events such as happenings in a pastoral scene. Thus, musical narration including symbols is closer to narration in verbal media types than to musical narration primarily based on icons.

Convention-based semiosis in instrumental music can be grounded not only on habits of representing certain existents and events but also on more specifically narrative conventions: habits of composing music that facilitate narration. Newcomb (1987) developed a rather general analogy between narration in written literature and instrumental music in terms of rule-governed, temporal perception of sensations representing events supported by narrative conventions that lead to certain expectations of what will follow, although these may or may not be fulfilled.

The above-mentioned researchers variously thought of represented events as actions or occurrences. Including notions such as actants and protagonists means that music is understood to represent conscious entities, minds, which act and thus create events in the form of actions. Consequently, it is possible but not necessary to think about musical events in terms of actions; also without represented minds that act there may be events in the form of occurrences. It must also be added that although the prerequisite for narration consists of representation of temporally interrelated events, the sometimes accented three-dimensionality of musical sounds also facilitate distinct spatial divisions of represented events: listening to musical sounds coming from different directions may trigger the creation of a virtual sphere containing spatially separated events. It is additionally clear that musically represented actions and occur- 
rences, distributed in time and possibly also in space, are generally more or less hierarchized by listeners: all changes of conditions are not considered to be equally important.

It goes without saying that collateral experience of the perceiver in the form of cognitive schemes and knowledge of earlier narratives may be vital for the realization of narration in instrumental music. This means that very simple verbal titles can also make event-structures of known narratives or cognitive schemes present to the mind of the perceiver; these eventstructures may then be realized or reinforced by the unfolding musical structure. Noting this, we are already on our way to explain narration in so-called program music, which includes more comprehensive verbal elements and hence falls beyond the borders of instrumental music as defined here.

Anyhow, perceiving music with or without verbal components involves striving toward grasping some sort of internal coherence. If one is aware of a title pointing toward something more concrete, one normally tries to incorporate this in the virtual sphere. If the piece of music only has an abstract title referring to musical form (like 'Piano Concerto No. 2') or no title at all, it may nevertheless not be self-evident how the parts belong together; it might be necessary to construe an overarching virtual narrator being responsible for, say, the representation of harsh clashes between existents and events that are best understood as ironic. Robert Hatten argued that narration in music includes some extreme contrasts in instrumental music that may be understood as shifts in the "level of discourse"; a sudden change in mood may place "all of the previous musical discourse in a new perspective" (Hatten 1991: 88). Avoiding the notion of discourse, I understand this as musical pieces presenting embedded virtual narrators introducing new and contrasting events or focalizing already represented events in a completely different way. Such ruptures may or may not be successfully reconciled by an overarching virtual narrator.

Narration in instrumental music can also be complex in other ways. There may be temporal differences between the complete narrative and the scaffolding core story, more specifically differences between the order, duration, and frequency of states and events in the narrative and the story (Genette 1980 [1972]: 33-160). This was investigated by Fred Everett Maus (1991) in reference to musical analyses made by other musicologists. Exemplifying differences in duration, Maus noted that a "musical tragedy" may be understood to have a story that lasts for months or years, while its complete narrative only lasts for eight minutes (Maus 1991: 31). 
Differences in order are illustrated with a 'cadential gesture', normally heard toward the end of a piece, instead being displayed early; one may then perceive a temporal disjunction between the temporal development of the story (which is concluded by the cadence) and the temporal development of the narrative (which presents the cadence at the outset) (Maus 1991: 28-30). Such a cadential gesture may actually be heard several times in the same piece, which may then be understood as appearing only once in the story but several times in the complete narrative; this would constitute a difference in frequency (Maus 1991: 30).

Musical narration, like all other forms of narration, may be perceived to be more or less truthful, and instrumental music is also qualified as a type of medium on the basis of, among other things, expected truthfulness. Again, it is important to note that instrumental music must be understood in terms of a variety of submedia (such as Indian instrumental ragas, progressive rock without song, or string quartets), so it is impossible to deliver anything but very general remarks here. Anyhow, it makes little sense to me to understand instrumental music (just as other forms of communication) in terms of fictionality. Although it cannot have the same kind of contiguity and refer to the same kind of indexical objects as certain other media types, it is very much connected to the perceived actual sphere. I suggest thinking in terms of mental contiguity, how things have actually been experienced by minds. Instrumental music is often expected to be, and indeed frequently is, truthful to objects such as motions, bodily experiences, emotions, and cognitive structures in general. Indexical objects like these are known to us not least through personal (external or internal) experiences and perceptions of both material and mental phenomena, so representations of them can be perceived to be more or less truthful. Because of our deeply shared resources of feeling and thinking, the indexical objects of music are often perceived to be highly universal.

\section{Mathematical Equations}

Compared to painting and instrumental music, mathematical equations constitute a quite different qualified media type that has hardly been researched in terms of narration. Nevertheless, it is a media type that illuminates the concept of narration in several ways. Although mathematicians may find some equations elegant, to some degree, they are not considered an art form, and compared to most other forms of communi- 
cation it is rather abstract; nevertheless, I believe its narrative capacity is striking.

Apart from figures representing numbers, mathematics is full of symbols that are rarely or never used outside this specific communicative domain. The basic feature of equations is that they state that two things are equal, which is symbolized by an equals sign $(=)$. Moreover, equations include variables, also called unknowns, that are often represented by letters such as $x, y$, and $z$. The point of equations is to communicate a mathematical problem that can be solved through calculation so that the two so-called expressions on each side of the equals sign can be demonstrated to be equivalent. This includes determining the values of the variables. The solution of the simple equation $2+x=5$ is that the variable $x$ stands for 3 because $2+3$ equals 5 .

Investigating the media modalities of mathematical equations makes it clear that the basic modes of equations are virtually identical to those of written verbal language. Hence, mathematical equations and written verbal language overlap smoothly in various ways and they can both efficiently be transmediated to spoken verbal language; that is, they can be read. Although both these qualified media types can, in principle, be realized in rather varied material and spatiotemporal settings, they normally appear on solid, inorganic, static, and two-dimensional surfaces. They are visual and their most salient signs are symbols: letters in the case of most Western languages, and, in the case of mathematics, figures and a range of specifically mathematical symbols (although there are also figures in language and letters in mathematics). Semiotically, however, written verbal language as well as mathematical equations may also be considerably iconic and hence semiotically multimodal. The equals sign in itself consists of two identical lines representing the absolute similarity of the two expressions in equations. The other mathematical symbols are placed on the surface in such a way that one may perceive similarities in gaps, distances, internal relationships, proportions, and other spatial qualities. The equivalence of the two expressions is represented iconically through their mirrored positions to the left and to the right, respectively, of the equals sign. Hence, the two expressions can be seen as signs for each other, so to speak.

We have noted that equations-like paintings, but unlike instrumental music-are non-temporal, which means that they do not absolutely effortlessly represent temporally ordered events. Unless one knows what an equation is and how to proceed in interpreting it, little significance will 
come out of it-and certainly not a virtual sphere containing temporally interrelated events. Furthermore, mathematical equations have evolved as media products in different cultures for thousands of years; therefore, as with other qualified media types, there is a historical dimension to consider. Like painting and instrumental music, equations do not constitute one single variation but rather a plenitude of submedia with intricate specific traits and widely different levels of complexity. Nevertheless, they are all expected to hold the specific communicative core quality of being tools for solving mathematical problems that may be more or less connected to understanding properties of the world.

Equations can represent existents that are not only numeral abstractions. Albert Einstein's famous formula $e=m c^{2}$ represents the interrelations among energy, mass, and the speed of light. However, these interrelations involve certain calculable relationships. Thus, equations in physics, chemistry, and engineering freely mix representations of existents that are anchored in the physical qualities of the world and mathematical existents such as numbers and proportions. Therefore, represented events in mathematical equations may be changes of conditions involving both more concrete and more abstract states.

A simple equation such as $1+1+4-5=x$ represents events understood as changes of numeral and proportional states. It can be read verbally as 'one plus one plus four minus five equals ex'. Whether the equation is read verbally or not does not affect its potential status as a narrative; either way, it represents a series of numeral changes-a succession of interrelated events. Nevertheless, a transmediation to a more elaborate verbal narrative might make the narrative core of the equation more visible: 'I was alone in the kitchen when my wife came home. Soon after that, also our four children arrived, but after a while I saw five family members go out in the garden. As I was very tired it was all very confusing: how many were actually left in the kitchen?'

One of the differences between the mathematical equation and its verbal transmediation, apart from the very concise narrative (consisting of virtually only a minimal core story) being expanded to a somewhat more elaborate narrative, is that events in the form of occurrences are changed into actions. Events represented by equations, if the latter are not embedded in other forms of communication, are normally occurrences rather than actions. Some events in equations have a larger impact (adding four rather than one is a more significant change of states), which means that they in a way can be hierarchized. In another way, however, all events are 
equally vital, as ignoring even the tiniest of them will invalidate any equation.

Mathematical narration in equations, involving represented events that are temporally interrelated in a meaningful way, is very much supported by conventions that facilitate narration. One such principal qualifying convention is, of course, the rule of sequential decoding. Like Western written verbal language, one must decode equations (roughly) from left to right and from top to bottom, in order to get the events in the right order (the represented events must be understood to follow the order of decoding the mathematical signs). There are many other conventions that I will not go into great detail with here. For instance, the use of parentheses determines how events are to be separated from each other and ordered. A section of an equation such as $3 y+7$ is totally different from $3(y+7)$. $3 y+7$ means that first the variable $y$ is tripled, and then seven is added to the product. On the other hand, $3(y+7)$ means that seven is added to the variable $y$, and then this sum is tripled. Because of the parenthesis convention, the two sections represent different events that are ordered in different ways and are therefore parts of different narratives. It can be concluded that represented events in equations are not only interrelated in general but temporally interrelated in specific ways.

Clearly, just as paintings or pieces of instrumental music, not all equations are best understood as narratives. A formula such as $e=m c^{2}$ is hardly a narrative. An equation such as $3 x+2^{2}+6-4=3 \times 4+3$, on the other hand, represents several interrelated events that change the developing numeral state: numbers are multiplied, squared, added, and subtracted. These calculations cannot easily be performed without collateral experience of numeral changes in one's life environment and, especially in the case of more complicated equations, mathematical training increasing one's ability to keep track of a multitude of intricately interrelated events. The assumption in every equation, including those that cannot be seen as narratives, is that they hold total internal coherence. Only when the balance between the two expressions is perfect, meaning that they represent exactly the same numeral state, can the equation be considered correctly formed or solved. Solving an equation involves strong mental indexicality: semiosis based on contiguity or real connections. This means that there are simply no options regarding how to connect the signs of the media product to what we think of as the mathematical reality. Signs such as $2^{2}$ are symbols, of course, but also indices to the extent that they put us in direct relation with general principles (in this case, a perceived actual exis- 
tence of the quality of twoness and the operation of multiplying a number with itself).

I also think that it is fruitful to make a distinction between narratives and stories in equations, at least to a certain extent. We have already stated that it is essential to decode the mathematical symbols in a specific order and that this order largely determines the order of represented events in the narrative. However, an important incentive for construing stories out of more extensive narratives is, we have also noted, to understand how the temporally interrelated events in the narrative would unfold if they would appear in directly perceived real life instead of being represented, and evoking such an incentive for equations seems almost nonsensical (unless, of course, they are embedded in other forms of communication representing more concrete aspects of the experienced world). This means that, normally, it would probably make little sense to think in terms of temporal differences (regarding order, duration, and frequency) between narrative and story in mathematical equations.

However, this does not eliminate the usefulness of the distinction between a complete narrative and a scaffolding core story, at least in equations that can be simplified. Isolating variables and simplifying equations are established ways of solving them step by step. For instance, $3 x+2^{2}+6-4=3 \times 4+3$ can initially be simplified so that $3 x+4+6-4=12+3$ remains. Simplifying it even further may lead to $3 x+10-4=15$. Perhaps this stage of the simplification could be considered the core story of the complete narrative. Further simplification leads to $3 x+6=15$, then $3 x=9$, and finally $x=3$, which is the solution-the final state after the events have passed by, so to speak.

However, I doubt the usefulness of the concepts of embedded virtual narrators and narratees for equations. The virtual spheres of mathematical equations are strictly regulated and can hardly allow for alternative perspectives. Furthermore, the focalization of the actual or overarching virtual narrator must be very strong to fulfill the firm requirements of equations. The expected truthfulness of the qualified medium of mathematical equations, as its internal coherence, is based on mental contiguity leading to indexical junctions: the logics of mathematics is not only valid within the virtual spheres of equations but also reflects conditions in the perceived actual sphere, including what we understand to be physical laws. Thus, the indexical objects in the perceived actual sphere may be both mental and material. As equations can be applied to both general phenomena and particular circumstances, the indexical objects in the perceived 
actual sphere may additionally be universal as well as particular, and wholes as well as details.

\section{Guided Tours}

The last qualified media type to be investigated is guided tours, which, to the best of my knowledge, have not yet been researched in terms of narration. This is a form of communication that may rely on a wealth of modes; hence, it exemplifies multimodal narration at its extreme. As a guided tour goes on, all forms of media products can be incorporated in a composite media product that integrates an increased amount of more or less dissimilar media types-together forming an amalgamated media type with its own character. Virtually all modes of the material, spatiotemporal, sensorial, and semiotic modalities may be included in guided tours, which entails that narration, and communication in general, can be realized through many different resources interacting in a multitude of complex ways that can only be hinted at here. In order to make the presentation somewhat more focused, I will mainly discuss one of many variations of guided tours: guided city tours.

All guided tours are temporal; they are not only perceived in time, which is the case for all media products, but, like music, they are unfolded in time. This is a convenient media trait for narration as it makes it possible to represent events in a certain order that cannot be escaped. However, guided tours can include parts that are static rather than temporal, although introduced in a temporal flow. As a guided city tour goes on, sculptures, reliefs, paintings, and other forms of visual, static, and twodimensional images are likely to appear. Also, various forms of inscriptions, signposts, and other static, visual, and verbal media products that must be sequentially decoded in order to make full meaning can be expected to be integrated. It is clear that these static incorporated media products may represent events that contribute to an overall narrative.

I would argue that guided tours are generally expected to be narrative. In other words: narration is one of the qualifying traits of guided tours. Most people who embark on a guided tour do not simply want to learn about a row of isolated states and events but also about their meaningful temporal interrelations. Spatial movement is another qualifying trait of guided tours. The participants in a guided city tour expect to be involved not only in a temporally evolving media product but also in a threedimensional, spatially evolving media product; they expect to walk or be 
driven around in the city-otherwise, it is simply not a tour. Furthermore, a tour cannot be considered a guided tour if it does not include a guide. Thus, a classic guided tour includes one or several corporeally present persons acting as guides, meaning that they are the actual narrators being present together with the participants, the actual narratees. Variations of guided tours, such as those involving audio guides (pre-recorded voices) instead of living persons, are not considered here.

As guided tours are composite media products potentially incorporating virtually all kinds of media products, they can represent practically all kinds of states and events, whether these events are actions or occurrences. These many forms of events may clearly be more or less strongly interrelated. It may be presumed that the actual narratees of guided city tours normally form a hierarchy of essential historical events, with certain major events at the top forming a scaffolding story: when the city was founded, when it became a capital, when it was invaded, when the most famous buildings were erected, when major parts of it were destroyed in a fire, and so on, and how these events are interrelated.

Given the open structure of guided tours, which normally include a broad variety of media products, probably often representing a multitude of also only vaguely interrelated events, actual narratees are likely to construe partly rather different hierarchies of events. Naturally, all forms of background knowledge of history, culture, geography, and so forth-and of earlier narratives including events related to these areas-facilitate the creation of relevant interconnections among the many directly represented events, as well as events that are not directly represented by the guided tour. Because most people expect guided tours to be narrative (they are often conventionally understood to constitute narratives), they are probably narrativized to a high degree in accordance with cognitive schemes related to historical, cultural, and political development.

However, diverging background knowledge among actual narratees may contribute to the formation of both differing stories and dissimilar hierarchies of events. Another factor that may add to a disintegration of stories construed by different perceivers is the complexity of representations in guided tours, meaning that there are no definite borders between a guided tour and everything surrounding it; almost any observation of the actual narratees may be incorporated into what they perceive to be the guided tour. In order to make sense of the sometimes scattered or even conflicting mass of represented events, either clearly within or in the border zone of the guided tour, the perceivers are likely to construe an over- 
arching virtual narrator that, to some extent, reconciles uncertainties and conflicts and pushes the many represented states and events toward internal coherence.

Probably much more often than not, guided tours harbor temporal differences between narrative and story (Genette 1980 [1972]: 33-160). It is difficult to arrange a guided city tour in such a way that the temporal order of the represented events in the complete narrative correspond precisely to the temporal order in the story. Moving through a city involves encountering quarters, buildings, squares, and monuments related to historical events that do not follow the path of the tour. Thus, the guide must, at least to some extent, jump back and forth among the historical events and still explain their interrelations so that a scaffolding story can be captured. Using spoken language, this is easily achieved though phrasings such as 'this happened long before ...' or 'this would lead to the events that I described earlier ...'. Differences in duration are also unavoidable. As a guided city tour only lasts for a few hours and the history of a city can be counted in decades, centuries, or even millennia, the duration of the narrative, directed by the actual duration of the media product, is very much shorter than the duration of the story-the duration of states and events as we assume they have appeared in real life. Differences in frequency are also ordinary elements in guided city tours. For instance, the most central events in the story, such as a collective action like a revolution or a natural occurrence like an earthquake, are likely to be represented several times in the narrative even though they occur only once in the story; this is modeled on our assumptions of real life events. In an abundantly multimodal media type such as guided tours, these repeated representations of the same events can be realized in a multitude of ways: through speech and gestures, sculptures, monuments, inscriptions, leaflets, engravings, paintings, ruins, or walls with cracks caused by the earthquake or bullet holes from the revolution.

It may not be self-evident how to understand the status of narrators in relation to the many media products that are integrated in a guided tour. However, it is clear that a guide is an actual narrator being present at the tour, using her own body and its extensions as media products while talking, gesticulating, and pointing, and also drawing objects in the surrounding into the realm of communication so that they act as media products. This presence enables two-way communication between the actual narrators and the actual narratees - the tour participants. There may also be communication among the actual narratees that adds to the guided tour. 
All this gives the participants the potential double role of actual communicators, possibly narrators, and actual narratees (in other words: they can interact with the guided tour). On top of this, the participants of a guided tour encounter various media products produced by other, often absent, actual narrators. Thus, one can say that a guided tour involves not only the actual narrators constituted by the present guides (and possibly the participants) but also a variety of mainly absent, living or dead actual narrators.

However, one could also argue that although the many media products in a guided tour are certainly directly perceived by the actual narratees, they are actually embedded in the overall narrative produced by the guides. Disregarding those many media products that are unavoidably haphazardly perceived by the participants, beyond the control of the guide, the media products that the guide actually incorporates in the tour (in a planned or improvised way) are not only perceived by the participants but in effect represented by the guide when somehow communicatively drawing the participants' attention to them. There is no conflict between something being simultaneously perceived and represented: the guide might say 'there you can see the castle' and at the same time point to it while the perceiver simultaneously actually looks at the castle, representing a certain event in political history. This means that while the architects, builders, and those who ordered the building of the castle are the initial actual communicators of, say, political power or cultural belonging, both these actual communicators and the media product-the castle-become embedded in the guided tour when represented by the guide. I believe that this view is feasible. Therefore, it is reasonable to say that whereas there are only a few present actual narrators of guided tours (the guides and possibly some participants), there are a multitude of embedded virtual narrators of embedded represented media products. In the end, it is the focalization of the actual narrators-what they know and what they choose to highlight about the city — that primarily determines how the narrative is formed.

Finally, it should be noted that the qualified medium of guided tours tangibly illuminates the relevance of truthfulness for narration. As many of the locations of the represented events in guided city tours are not only represented but also actually perceived during the tour, the door is open for media products to have direct and strong interactional contiguity with the represented states and events in the narrative. There is an important difference between someone saying that a certain city has a castle bearing 
material witness to both an earthquake and a revolution, and someone saying that the castle in front of you has traces of these dramatic events. Directly perceiving a building with cracks and holes, which could have resulted from an earthquake and battles, may add a vital indexical dimension: the participants of the guided tour directly perceive material objects that are drawn into the communication and thus acquire the function of media products; media products that are really connected to the perceived actual sphere.

Therefore, in contrast to the previously discussed qualified media types, guided city tours provide opportunities for not only mental but also material contiguity between representamens of the media product and objects in the perceived actual sphere. While in the case of cracks and bullet holes the perceived contiguity is mechanical, also electromagnetic, chemical, organic, and other forms of material contiguity may be involved in achieving external truthfulness in guided tours. Grossly simplifying the issue of indexical object in guided tours, it can be surmised that participants of city tours expect them to be truthful to both material and mental objects, to both details and wholes, to objects that are manifested previously and currently rather than subsequently (to history rather than future), and to particulars rather than universals (to one specific city rather than all cities). Additionally, it is generally both accepted and perhaps even expected that guided city tours are truthful to other virtual spheres; that is, to earlier communication regarding the city in question, including accepted history writing but also legends, fanciful tales, and other submedia types that are less directly anchored in the perceived actual sphere.

\section{Rounding OFF}

I will stop here before my account of transmedial narration becomes too repetitive. More and lengthier examples of qualified media types would no doubt highlight additional media-specific intricacies, but I doubt that the essential transmedial principles would be further clarified. However, the conceptual tools that I have offered in this treatise for understanding and analyzing narration should be useful for disentangling the narrative potential of any form of communication. They should also, hopefully, be helpful for perceiving both media similarities and media differences that facilitate or obstruct transmediation of narratives among different forms of basic and qualified media types. 


\section{REFERENCES}

Elleström, Lars. 2017. Bridging the gap between image and metaphor through cross-modal iconicity: An interdisciplinary model. Iconicity in Language and Literature 15: 167-190.

Genette, Gérard. 1980 [1972]. Narrative Discourse: An Essay in Method. Translated by Jane E. Lewin. Ithaca, NY: Cornell University Press.

Grabócz, Márta. 2008. Classical narratology and narrative analysis in music. In $A$ Sounding of Signs: Modalities and Moments in Music, Culture, and Philosophy, ed. Robert S. Hatten, Pirjo Kukkonen, Richard Littlefield, Harri Veivo, and Irma Vierimaa, 19-42. Imatra: International Semiotics Institute.

Hatten, Robert. 1991. On narrativity in music: Expressive genres and levels of discourse in Beethoven. Indiana Theory Review 12: 75-98.

Kafalenos, Emma. 1996. Implications of narrative in painting and photography. New Novel Review 3: 53-64.

Kibédi Varga, A. 1988. Stories told by pictures. Style 22: 194-208.

Maus, Fred Everett. 1991. Music as narrative. Indiana Theory Review 12: 1-34.

McClary, Susan. 1994. Narratives of bourgeois subjectivity in Mozart's Prague Symphonie. In Understanding Narrative, ed. James Phelan and Peter J. Rabinowitz, 65-98. Columbus: Ohio State University Press.

- 1997. The impromptu that trod on a loaf: Or how music tells stories. Narrative 5: 20-35.

Micznik, Vera. 2001. Music and narrative revisited: Degrees of narrativity in Beethoven and Mahler. Journal of the Royal Musical Association 126: 193-249.

Newcomb, Anthony. 1987. Schumann and late eighteenth-century narrative strategies. 19th-Century Music 11: 164-174.

Pawlowska, Malgorzata. 2014. Musical narratology: An outline. In Beyond Classical Narration: Transmedial and Unnatural Challenges, ed. Jan Alber and Per Krogh Hansen, 197-220. Berlin and Boston: De Gruyter.

Seaton, Douglas. 2005. Narrative in music: The case of Beethoven's 'Tempest' sonata. In Narratology Beyond Literary Criticism: Mediality, Disciplinarity, ed. Jan Christoph Meister, Tom Kindt, and Wilhelm Schernus, 65-81. Berlin and New York: Walter De Gruyter.

Tarasti, Eero. 1994. A Theory of Musical Semiotics. Bloomington: Indiana University Press.

Weitzmann, Kurt. 1947. Illustrations in Roll and Codex: A Study of the Origin and Method of Text Illustration. Princeton, NJ: Princeton University Press.

Wickhoff, Franz. 1895. Der Stil der Genesisbilder und die Geschichte seiner Entwicklung. In Die Wiener Genesis, ed. Wilhelm Ritter von Härtel and Franz Wickhoff, vol. 2, 1-96. Vienna: F. Tempsky.

Wolf, Werner. 2003. Narrative and narrativity: A narratological reconceptualization and its applicability to the visual arts. Word o Image 19: 180-197. 
Open Access This chapter is licensed under the terms of the Creative Commons Attribution 4.0 International License (http://creativecommons.org/licenses/ by $/ 4.0 /$ ), which permits use, sharing, adaptation, distribution and reproduction in any medium or format, as long as you give appropriate credit to the original author(s) and the source, provide a link to the Creative Commons licence and indicate if changes were made.

The images or other third party material in this chapter are included in the chapter's Creative Commons licence, unless indicated otherwise in a credit line to the material. If material is not included in the chapter's Creative Commons licence and your intended use is not permitted by statutory regulation or exceeds the permitted use, you will need to obtain permission directly from the copyright holder.

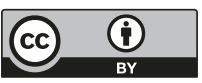

